\title{
Improvement and Exploration of Teaching Method of Fluid Power Transmission
}

\author{
Jungong $\mathrm{Ma}^{*}$, Wanguo Li, Juan Chen, Jian Fu, Xiangdong Wu, Weidong Li, Bao \\ Meng, Jianbin Zhang, Min Wan, Yongling Fu
}

School of Mechanical Engineering and Automation, Beihang University, Beijing, China

${ }^{*}$ Corresponding author. Email: jgma@buaa.edu.cn

\begin{abstract}
The fluid power transmission is the core course of the mechanical engineering discipline, characterized with the width of application scope and depth of knowledge and technology innovation. It covers much professional knowledge, involves certain theoretical basis and has strong practicality. It has been very difficult for many students to learn and understand some of the hydraulic components and hydraulic systems. At the same time, due to the compression of teaching hours, we are faced with the contradiction of complicated teaching content, more teaching difficult and less teaching hours, as well as the disconnection between theory and practice. Through combinating different reformed method of Flipped Classroom, Ethical Functions, stereoscopic teaching method, teaching model,building mind maps and so on, the construction of curriculum information and the application of modern education technology can be accelarated. It is a process of finding optimized teaching methods of fluid transmission course, and of the cultivation of innovative talents. The exploration is aiming to comprehensively improve the students' independent learning power, in-depth analysis ability and the spirit of innovative design.
\end{abstract}

Keywords: Fluid power transmission, Curriculum teaching, The teaching reform.

\section{《流体传动》教学方法改进与探索}

马俊功*, 李万国, 陈娟, 付剑, 吴向东, 李卫东, 孟宝, 张建斌, 万敏, 付永领

北京航空航天大学机械工程及自动化学院, 北京, 中国

*通讯作者. 邮箱: jgma@buaa. edu. cn

\section{中文摘要}

《流体传动》是机械工程学科的核心专业课程, 具有知识的应用广度和技术的创新深度, 它覆盖的 专业知识较多, 既涉及一定的理论基础, 又具有较强的实践性, 许多学生学起来有较大难度, 对于 一些液压元件及液压系统难以理解; 同时由于课时压缩又面临教学内容多、教学难度大和教学课时 少的矛盾，以及理论和实践脱节等问题。通过翻转课堂、删繁就简、立体教学、使用教具、构建思 维导图等多种教学手段的改革，加快课程信息化建设和现代教育技术应用，研究适合创新型人才培 养的《流体传动》课程教学方式方法, 全面提高学生的自主学习动力、深度分析能力和创新设计的 精神。

关键词: 流体传动; 课程教学; 教学改革. 


\section{1. 引言}

从蓝天到水下，从军用到民用，从重工业到轻工 业，到处都有流体传动技术的应用场景，很多场合都 发挥着不可替代的作用。《流体传动》以流体力学为 理论基础，围绕机械专业的工程实际，探究液压与气 动技术一般规律及其具体应用的课程，重点突出基础 性、实践性、综合性。主要内容包括: 流体力学基本 理论、液气压元件及基本回路、比例伺服技术和典型 液气压传动系统等。传统教学手段以灌输为主, 难以 激发学生的积极性和主动性, 教学效果欠佳。探索教 学方式和方法的变革, 在充分利用多媒体教学设备和 教学课件的基础上, 通过对课程的重点和难点进行剖 析, 合理划分线上线下教学内容, 优化教学方式和手 段, 进而激发学生主动学习知识的兴趣, 引导学生思 考和探究, 锻炼学生们独立分析和解决实际问题的能 力, 是本门课程迫切需要解决的问题。

\section{2. 问题与不足}

(1) 教学内容的广度和深度都有待拓展, 与前后 课程的联结不够紧密, 特色不够鲜明;

（2）目前的教学方法主要集中在以课件形式为主 的线上授课, 教学方式单一, 缺乏使用数字化教学工 具, 缺少多样化的教学手段, 学生掌握的知识点仍停 留在书本知识层面, 教学效果较差。教学方式与对课 程高阶性、创新性方面的要求存在差距;

（3）网络资源的开发运用还不够充分; 在教学中 没有应用到流体传动方面的仿真软件, 不利于学生掌 握最新的专业工具及技术;

(4) 目前的教学目标测量的主要手段是通过作业 与考试的形式, 集中在对基础知识的掌握程度, 缺乏 从研究性、创新性、综合性内容的角度来衡量学生对 知识点的掌握情况, 以及缺少以激发学习动力和专业 志趣为着力点完善过程评价制度, 学生学习本课程的 动力不足, 课堂外的学习时间偏少;

（5）课程建设、课程管理和教学团队建设方面的 投入不够, 教学团队如何有效协同和发挥教学团队的 效果需要改进和提升。

\section{3. 改革思路}

（1）教学内容按照必需 $\rightarrow$ 够用 $\rightarrow$ 前沿的原则, 提 高理论深度、加大技术应用的广度, 强化实践技能、 工程能力和科研能力的训练, 突出航空航天特色, 紧 密与前后课程的支撑和联结作用;

（2）在线上和线下教学等各个环节形成有效协同 机制; 建立知识体系、教学资源与工具、学生课外练 习与实践、综合考评等各个环节有机统一的教学模式; 鼓励学生进行探索式学习, 提升课程学习的挑战性,
促进知识点的融会贯通, 提高学生对基础理论知识进 行应用和解决复杂工程问题的能力;

（3）集中人力和物力建设线上和线下教学网络资 源和仿真软件平台, 为学生提供个性化的学习资源与 学习路径、提供方便的仿真软件学习和使用环境。

（4）在教学成效评估方面, 基于课程知识点, 加 强研究型、项目式学习, 丰富探究式、论文式、报告 答辩式等作业评价方式, 提升课程学习的深度; 探索 非标准化、综合性等评价方法; 建立学生学习效果检 验、跟踪与反馈有效运行与管理方法;

（5）建立有经验教师对青年教师传帮带和定期进 行教学经验交流等的教学素养提升机制, 定期发布教 学总结报告, 充分发挥名师在课程教学中的示范作用, 推动教师队伍的整体教学水平的提高。

该模板在MS Word 2007中进行了修改, 并保存为 $\mathrm{PC}$ 的“Word 97-2003文档”, 为作者提供了准备论文电 子版所需的大多数格式规范。已指定所有标准纸张组 件的原因有以下三个:（1）格式化单个纸张时易于 使用; (2) 自动符合电子要求, 从而有利于同时或 以后生产电子产品；（3）整个产品风格的一致性 会 议记录。

\section{4. 教学方法改进}

注重教学方法改革、加快课程信息化建设和现代 教育技术应用。树立了以学生为主体, 以教师为主导 的教育理念, 组织教学, 将传授知识与培养人才有机 结合、注重培养学生创新精神和个性发展, 在课堂教 学中普遍采用启发式和互动式的教学方法, 翻转课堂 的教学形式, 提高教学效果。

\section{1. 线上线下混合式教学, 提高课堂效益}

应用 “翻转课堂” 实现混合式教学, 一方面解决 学时少与内容多之间矛盾, 另一方面, 给学生更多自 主学习时间, 提高他们的积极性, 回归到教学的本质: 学生为中心, 教师为主导, 实现教学相长。

对基本原理、基本结构等基础知识方面的内容, 以利用网络或移动在线学习平台让学生课前通过自 主学习的方式进行学习为主, 如流体静力学、液压元 件、气动元件、液压辅件、气动辅件的工作原理、特 点等内容, 课上教师主要讲解共性难点问题。而对于 流体动力学、液压系统、液压伺服系统、气动系统基 本回路及其在典型设备中的应用，以及液压系统及气 动系统的基本设计方法等内容, 则以线上学习为辅, 在课堂上进行案例教学和项目教学以及分组讨论探 究式学习为主, 以此巩固和提升学生对知识的应用能 力, 并且线上线下学习的过程要进行评估考核, 要形 成配套的考核监督体系。 


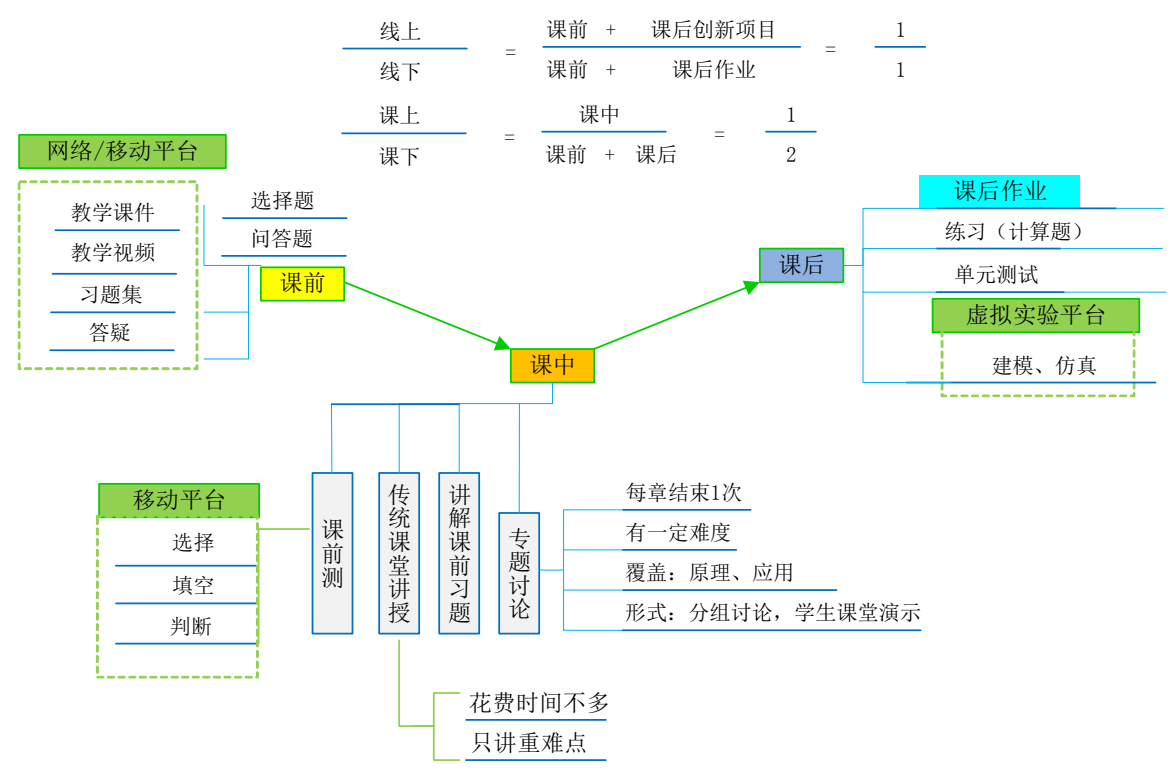

图1 翻转课堂及学时分配

\section{2. 删繁就简式讲解, 解决主要矛盾}

《流体传动》知识体系复杂，包括较多内容，在 具体教学中, 可以删繁就简, 对复杂内容简化处理, 根据章节标题建立整体的知识体系结构, 对每个章节 中的重点问题进行集中讲解, 然后举一反三引导学生
的联接性思考。例如对于泵结构形式讲解，可以讲解 单柱塞液压洜工作原理, 启发学生根据工作流程推导 结构。学生分析叶片原和齿轮洜性能、结构后, 找出 异同点, 可进行综合性的比较、分析, 从而更加清晰 的掌握相关内容。
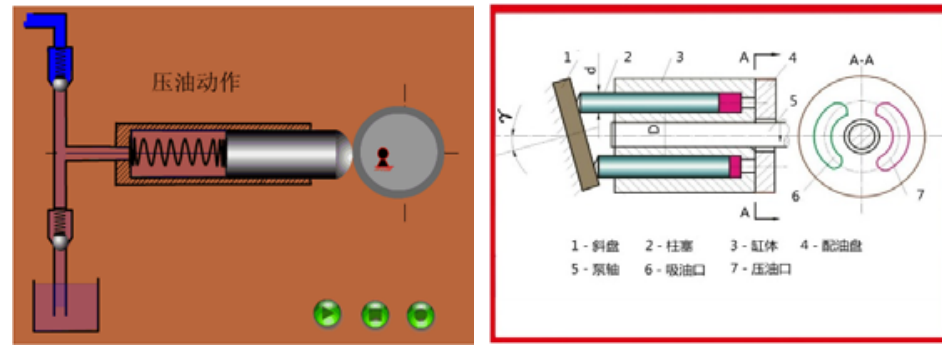

(b)

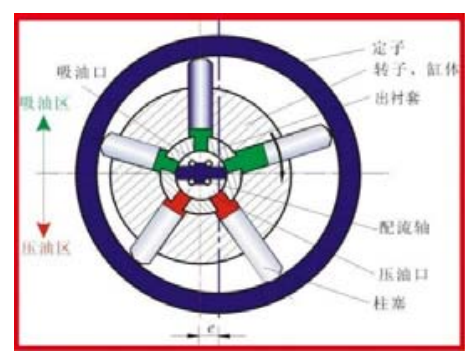

(c)

（a）容积式原 （b）轴向柱塞葲 （c）径向柱塞㫤

图2 容积式液压㫤工作原理

\section{3. 充分运用现代手段, 实现立体教学}

充分使用现代技术和新设备, 实现多媒体教学, 对课本中较为抽象的问题以更加生动、形象的形式展 现出来。多媒体教学可以为学生搜集文字、图片、影 像、音频等相关教学资源。

例如对于液压元件结构特点和工作原理部分的讲 解, 使用多媒体设备可以制作电子课件。以Solidworks 等软件设计元件的模型, 从三维立体式角度为学生展 示液压/气动元件的外部造型和内部结构; 以Flash的 软件构建一个合适的液压/气动系统, 以动画形式向学
生展示不同液压/气动元件在工作时, 各元件可动部分 的移动状态变化, 并展示液/气流在不同控制回路中的 流动过程; 以FluidSIM仿真软件搭建相应的系统，实 时显示系统内各元件性能参数的动态变化过程, 以及 敏感参数对系统性能的影响, 这样就将元件和系统也 结合在一起，建立了元件和系统的有机联系。

运用多种技术手段讲解《流体传动》知识, 具有 直观、清晰的特点, 可以实现三维模型-- $\rightarrow$ 二维原理 $-\rightarrow$ 实时动态性, 元件 $-\rightarrow$ 系统两个层次的展示, 使 学生能够更加全面了解相关原理, 学习兴趣和探究欲 望被充分调动，继而激发起创新思维去思考相关问题。 


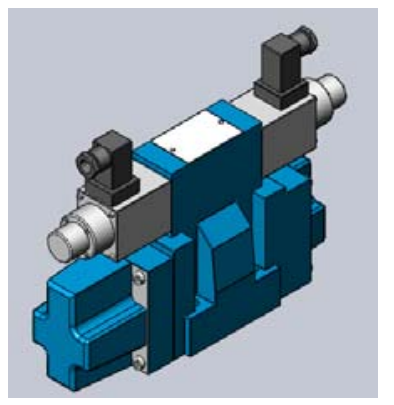

(a)

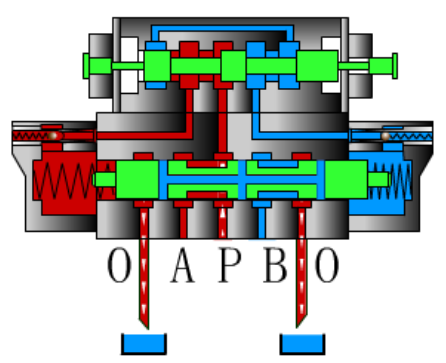

(b)

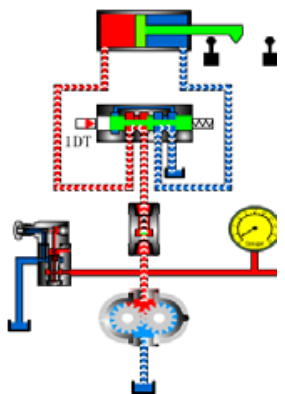

(c)

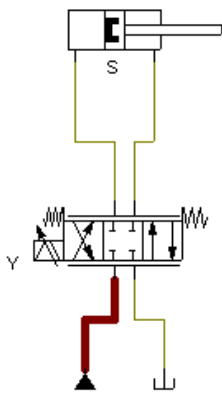

(d)
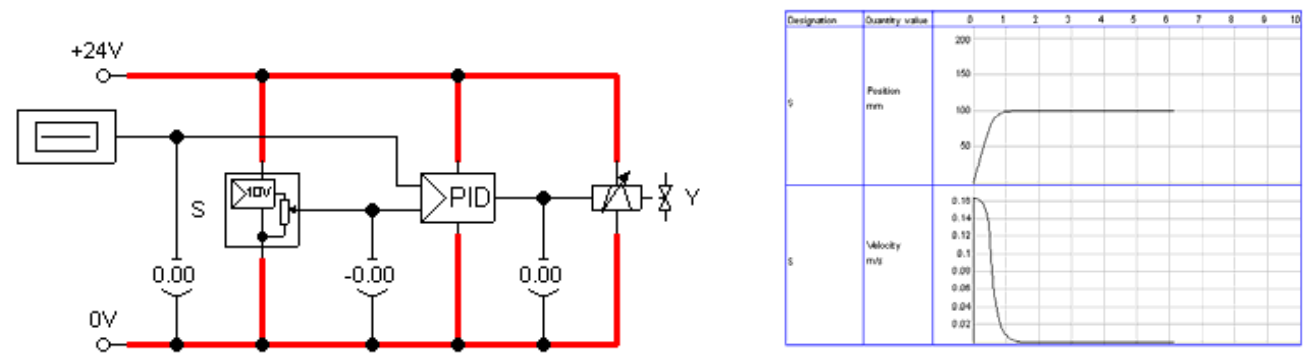

(e)
(a) 比例换向阀三维图
(b) 比例换向阀原理图
(c) 比例阀控缸动画图
(d) 比例阀控缸原理图 (e) PID控制闭环回路 (f) 液压缸位移及速度曲线

图3 比例换向阀及其控制系统特性

\section{4. 适当使用教具, 增强感性认知}

对于比较复杂的液压元件的讲解, 可通过使用教 具的方式对学生进行演示, 使学生有更直观的理解。
例如在讲解齿轮葲的过程中, 可通过对液压元件进行 拆装, 使学生了解其结构及工作原理。同时采用分组 教学的方法, 使每一位同学对元件进行拆装, 以便于 更好地掌握课程内容。
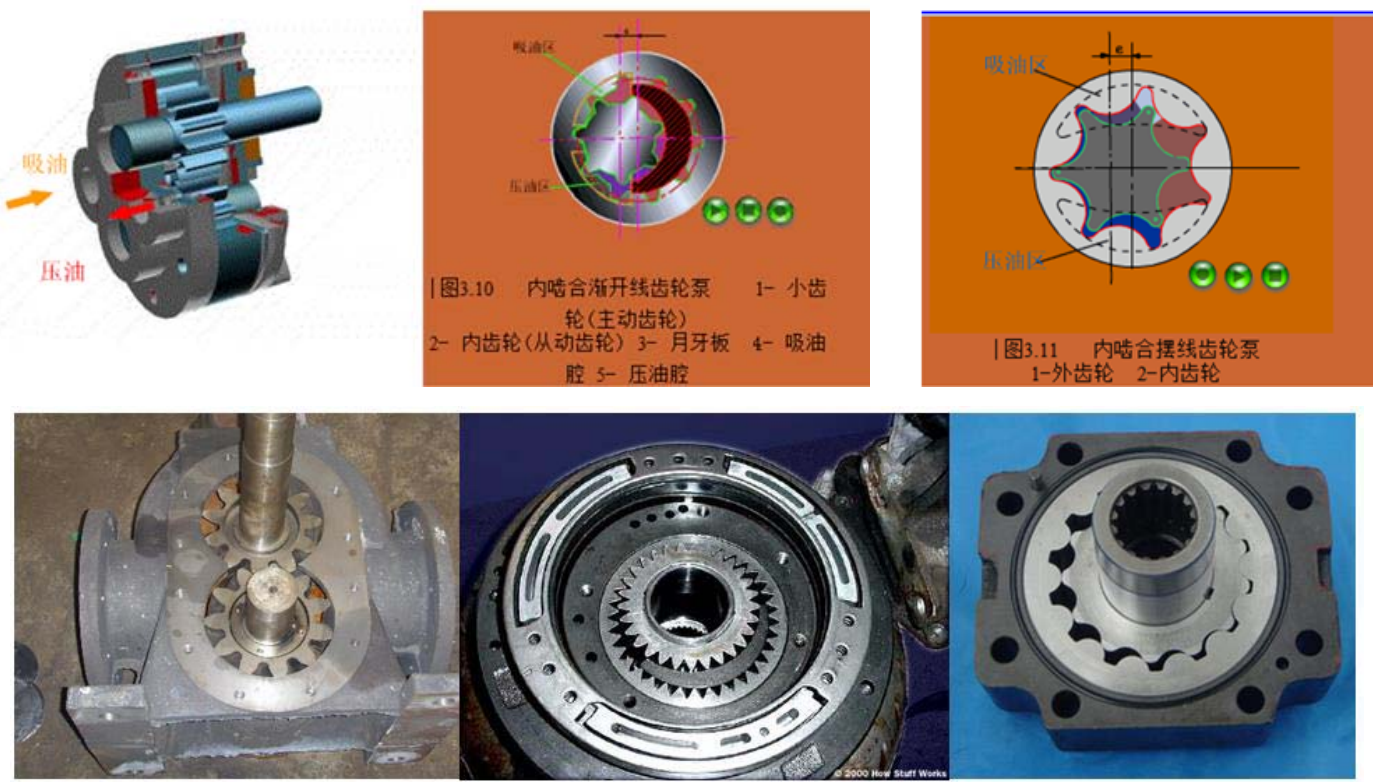

图4 齿轮原教具图

\section{2. 构建思维导图, 提开抽象思维能力}

学生在专业课程的学习中不仅需要掌握书本理论 知识, 还要有意识的培养自己的学习思维, 老师在讲 解《流体传动》内容的过程中, 也可以应用思维导图
教学法, 帮助学生构建基础的理论体系, 使学生在大 脑中形成体系化的知识学习体系。使用思维导图是帮 助学生克服思维障碍, 老师对不同单元中的重点知识 进行提取, 构建出一个基础的知识网络, 在知识网络 的不同结构 “树” 中, 补充探索活动或实验活动, 让 
学生对知识网络结构树中的不同知识点层层探索、验 连接起来, 可以获得系统的学习并提升抽象思维能力。 证, 并对各个关键词、知识点, 用色彩、图像等辐射

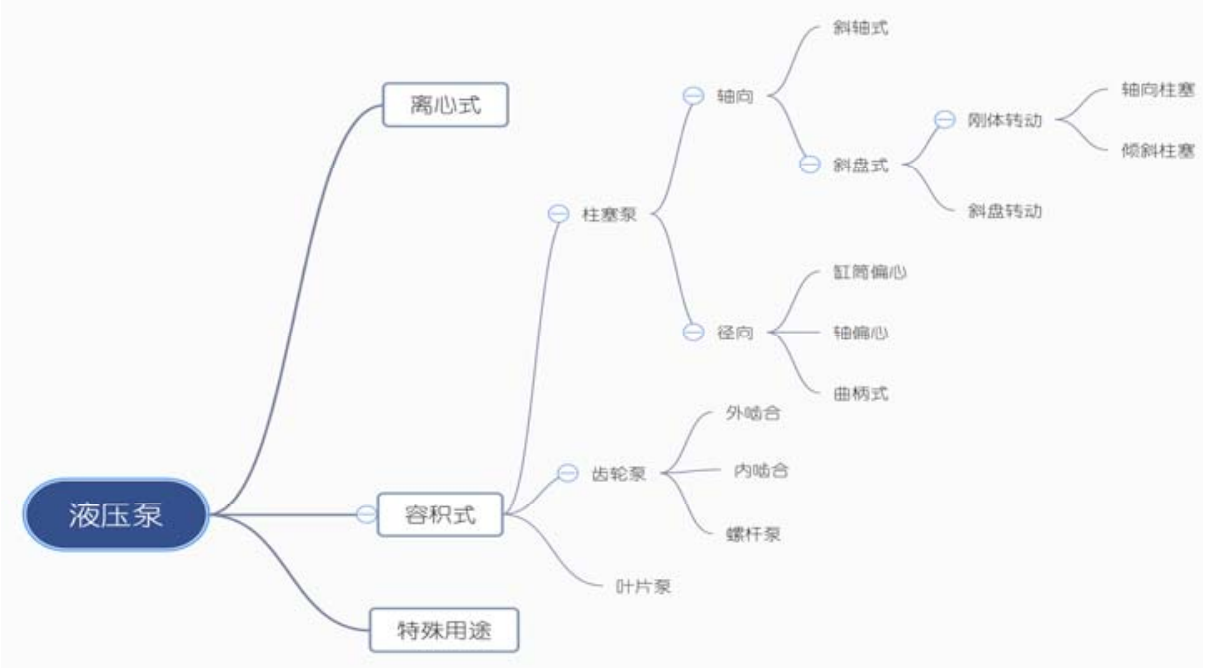

图5 液压泵分类

\section{5. 结束语}

流体传动技术是一个国家工业发展水平的重要标 志之一，现已大量应用于工业领域，在很多方面改变 了人类的生活。因此作为机械专业的教师必须重视这 门课程的教学, 在教学过程中改变传统的教学模式, 采用多种教学方法和教学手段, 并采用理实一体化教 学, 利于学生学习此类知识效率与质量的提高。为此, 要不断加强对此类课程的研究, 注重课堂中知识的传 授与实践的落实以及学生创新思维的培养, 同时尽可 能利用信息技术构建网络教学平台, 促进流体传动授 课质量的提高。近几年, 通过《流体传动》课程全方 位的教学改革与实践, 学生反应、学校评价都很好。

\section{致谢}

感谢北京航空航天大学对《流体传动》课程的教 改支持。

\section{REFERENCES}

[1] Zhengtao Liu, Qianxin Wang, Linping $\mathrm{Xu}$, Discussion on the Construction and Implementation of Online and Offline Mixed "Gold Course" in Applied Undergraduate Colleges, Jiangsu Higher Education, No.11, 2020, pp. 80-83.

[2] Shaogang $\mathrm{Hu}$, Jianfang Xu, Xiaofeng Wu, "Practice of Construction of Excellent Course for Hydraulic and Pneumatic Transmission", Journal of Changzhou Institute of Technology, No.3, 2014, pp. 78-81.

[3] Cuiting Huang, Jian Lv, Research on the Reform of Mixed Teaching of Tourism Policies and Regulations in Application-oriented Universities under the Background of "Internet +", Science and Education Guide - Electronic Edition (Middle Edition), No.1,2021, pp. 78-81. 